$\begin{array}{ll} & \text { Etnográfica } \\ \text { etnográfica } & \text { Revista do Centro em Rede de Investigação em }\end{array}$

Antropologia

vol. $24(2) \mid 2020$

Vol. $24(2)$

\title{
Introducción al dossier: heterogeneidad ontológica y ontologías en conflicto en Sudamérica
}

Introduction: ontological diversity and conflicting ontologies in South America

\section{Florencia Tola}

\section{OpenEdition}

\section{Journals}

Edición electrónica

URL: https://journals.openedition.org/etnografica/9013

DOI: 10.4000/etnografica.9013

ISSN: 2182-2891

\section{Editor}

Centro em Rede de Investigação em Antropologia

\section{Edición impresa}

Fecha de publicación: 1 junio 2020

Paginación: 455-464

ISSN: 0873-6561

\section{Referencia electrónica}

Florencia Tola, «Introducción al dossier: heterogeneidad ontológica y ontologías en conflicto en Sudamérica », Etnográfica [En línea], vol. 24 (2) | 2020, Publicado el 31 julio 2020, consultado el 20 enero 2022. URL: http://journals.openedition.org/etnografica/9013 ; DOI: https://doi.org/10.4000/ etnografica. 9013

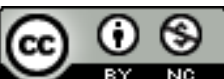

Etnográfica is licensed under a Creative Commons Attribution-NonCommercial 4.0 International License. 


\section{Introducción al dossier: heterogeneidad ontológica y ontologías en conflicto en Sudamérica}

\section{Florencia Tola}

En Latinoamérica, como en otras partes del mundo, los pueblos indígenas se hallan atravesados por el despojo territorial y la violencia sistemática por parte de los Estados y las empresas privadas. En este escenario, el estudio de las ontologías no significa un desinterés por la dimensión política que viven dichos pueblos, sino que, por el contrario, implica, entre otras cosas, buscar nuevos modos de acercarse a ésta. Para comprender las relaciones entre los pueblos indígenas y los Estados, el acercamiento a los conflictos y la heterogeneidad ontológicos permite echar luz sobre los equívocos que estas relaciones generan. El territorio, la política y los recursos no remiten necesariamente a lo mismo para los Estados, las multinacionales y los pueblos indígenas. En este sentido, la ontología no tiene tanto que ver con una pluralidad de perspectivas culturales, sino con una multiplicidad de mundos. Tomar en serio otras maneras de componer el mundo es reconocer que no nos encontramos en el terreno de las epistemologías nativas, sino de las ontologías, y las ideas expresadas por nuestros interlocutores dejan de ser leídas en términos de imaginación cultural, metáforas o símbolos de otra cosa.

PALABRAS CLAVE: ontologías, conflictos ontológicos, cosmopolítica.

Introduction: ontological diversity and conflicting ontologies in South America - In Latin America, as in other regions of the world, Indigenous Peoples suffer territorial dispossession and systematic violence by states and private companies. In this context, the study of ontologies does not mean a lack of interest in the political dimension that these peoples experience. On the contrary, it implies, among other things, seeking new ways of approaching politics. In order to understand the relationships between Indigenous Peoples and National states, the approach on conflicts and ontological heterogeneity allows to highlight the misunderstandings that these relationships involves. Territory, politics and natural resources do not refer to the same "thing" for National states, multinationals enterprise and Indigenous Peoples. In this sense, ontology has less to do with a plurality of cultural perspectives, but with a multiplicity of worlds. To take other forms of composing the world seriously is to recognize that we are not in the domain of native epistemologies, but of ontologies, and the ideas expressed by our interlocutors can no longer be read in terms of cultural imagination, metaphors or symbols of the reality out there.

KEYWORDS: ontologies, ontological conflicts, cosmopolitics.

TOLA, Florencia (tolatoba2015@gmail.com) - Conicet, Argentina. 
EL PROPÓSITO DE ESTE DOSSIER ES INDAGAR, A PARTIR DE ACERCAMIENtos etnográficos, en algunos aspectos que, desde hace algunas décadas, son centrales de la antropología en general y de aquella relativa a pueblos indígenas en particular. Por un lado, todos los artículos aquí reunidos exploran las ventajas de una antropología ontológicamente orientada respecto de un estudio de las representaciones culturales. ${ }^{1}$ Por otro, se preguntan por las potencialidades de perspectivas y metodologías ontológicas cuando se trata de abordar situaciones de conflictos y equivocaciones interétnicas y reivindicaciones indígenas de diverso tipo. En Latinoamérica, como en otras partes del mundo, los pueblos indígenas se hallan atravesados por el despojo territorial y la violencia sistemática por parte de los Estados y las empresas privadas. En este escenario, tal como los diversos artículos que constituyen este volumen lo muestran, el estudio de las ontologías no significa un desinterés por la dimensión política que viven dichos pueblos, sino que, por el contrario, implica, entre otras cosas, buscar nuevos modos de acercarse a ésta.

Como es sabido, el denominado "giro ontológico" se erige sobre replanteos conceptuales centrales de la antropología (como la oposición naturaleza/cultura, entre otros), reacciona frente al Antropoceno y constituye una "renovadora política de la naturaleza" (Charbonnier, Salmon y Skafish 2017: 9) en tanto implica una revisión profunda de los principios ontológicos que guían "la organización política y material de la modernidad" (2017: 8). Este giro no se limita, entonces, ni a ser una mera inquietud intelectual, ni a presentarse como una corriente desarrollada sobre el telón de fondo de la crisis ambiental. En América Latina, en las últimas décadas se han visto intensificados procesos económicos y políticos que, bajo el supuesto énfasis en el desarrollo y la inserción de las economías locales en los mercados internacionales, terminaron por agudizar las desigualdades y provocaron despojos territoriales, la degradación ambiental y conflictos sociales de diverso tipo. Para dar un ejemplo, el Gran Chaco sudamericano ${ }^{2}$ ha sufrido un proceso creciente de expansión de la frontera agropecuaria y se ha convertido en una zona importante de plantación de soja destinada a la exportación. Estos fenómenos, motivados por el progreso

1 Teorizaciones en relación a las diferencias entre ambos tipos de abordajes pueden encontrarse en Descola (2005, 2010, 2014), Henare, Holbraad y Wastell (2007), Venkatesan (2010), Holbraad (2012), Holbraad, Pedersen y Castro (2014), Charbonnier, Salmon y Skafish (2017), entre otros.

2 El Gran Chaco es el tercer gran territorio biogeográfico y morfoestructural de América Latina después del Amazonas y el Sistema Sabánico Sudamericano, y el segundo en superficie cubierta por bosques después de las selvas pluviales tropicales del Amazonas y del Pacífico colombo-ecuatorianas. Ocupa más de $1.000 .000 \mathrm{~km}^{2}$ y se extiende a lo largo de cuatro países (Argentina, Bolivia, Paraguay y Brasil). Esta región se halla delimitada por los ríos Paraná y Paraguay hacia el este, por la precordillera de los Andes hacia el oeste, por los llanos de Chiquitos y la meseta del Mato Grosso hacia el norte y por la cuenca del río Salado hacia el sur. En la Argentina, la región chaqueña se ubica en varias provincias del centro y norte del país. 
regional y nacional, fueron concomitantes con la creciente deforestación, la alteración del ecosistema, la migración de población rural y los intentos de desalojo de comunidades indígenas (cf. De la Cruz 2004; Iñigo Carrera 2012). Debido a la expansión del cultivo de soja y de la ganadería, al incremento de las áreas deforestadas, a la explotación de hidrocarburos, a la contaminación de los ríos, esta región sufre, al igual que tantas otras, procesos de desertificación y depredación de sus recursos naturales (commodities) que afectan la vida de los pueblos indígenas. De hecho, muchas de las comunidades indígenas fueron privadas de sus territorios, vieron alteradas sus prácticas de subsistencia, sufrieron desalojos y atropellos dentro de sus predios comunitarios, en fin, han visto vulnerados todos sus derechos colectivos, entre ellos, los territoriales.

A pesar de que el giro ontológico no constituya una corriente homogénea ni todos los antropólogos adopten una misma definición de ontología, varios de los trabajos de esta línea, preocupados, como hemos dicho, por las derivas de las políticas de la naturaleza imperantes hasta el momento, coinciden en la importancia de tener en cuenta a más-que-humanos (more-than-human), no-humanos, híbridos y multisespecies, en una tendencia que ha sido considerada posthumanista ( $c f$. Kohn 2012, 2013). De hecho, el cuestionamiento de la Gran División naturaleza/cultura como matriz universal y las etnografías que mostraron el papel central de la relación con los no-humanos en otras ontologías (amerindias, aunque no solo), dieron lugar a estudios sobre lo que Bruno Latour (1991) denominó "naturalizaciones alternativas", es decir, modos de concebir la naturaleza que no la reducen a su relación con los humanos ni la explican necesariamente en términos antropomórficos. Latour se interesó por la constitución ontológica moderna y mostró que las distinciones entre objetos y sujetos y humanos y no-humanos son propias del pensamiento euro-americano y que, por tanto, es posible generar en diálogo con otros pensamientos una nueva constitución que reensamble aquello que la Gran División había separado. El ejercicio de ontología estructural llevado a cabo por Philippe Descola (2005) también apunta en una dirección similar. Su modelo sobre las diversas ontologías a las que definió como "modos de identificación" entre humanos y no-humanos a partir de rasgos de interioridad y de fisicalidad muestra que la occidental y naturalista es una entre otras opciones disponibles.

En las investigaciones de otros antropólogos el giro ontológico cobró características diferentes. Entre un grupo de académicos ingleses influenciados por la propuesta del brasileño Eduardo Viveiros de Castro en relación al perspectivismo amerindio, por ejemplo, se constituyó como un proyecto de renovación metodológica. Ellos impulsaron el desarrollo de una antropología recursiva en un intento que fue incluso considerado como un modo de "perspectivizar" la disciplina y que condujo a Martin Holbraad a elaborar su método ontográfico. Por su parte, en la antropología norteamericana y canadiense las derivas del giro ontológico cobraron un matiz marcadamente político al nutrirse de los 
debates no solo sobre ontologías sino también sobre ecología, economía política y decolonialidad. La propuesta de investigación-acción llevada adelante por algunos de estos antropólogos se basó, entre otras cosas, en dar cuenta de los conflictos que emergen allí donde diversos mundos entran en contacto en pos de la constitución de un pluriverso que los incluya a todos.

El presente dossier retoma algunos de los postulados de este giro disciplinar con el fin de profundizar en dos aspectos que, en los contextos indígenas, resultan de fundamental importancia: la heterogeneidad o pluralismo ontológico y los conflictos que dicho pluralismo conlleva. Más que considerar a las ontologías de modo estático, homogéneo o a-histórico, Descola $(2005,2014)$ demostró la necesidad de dar cuenta de las transiciones entre ontologías y las "hibridaciones progresivas" que se producen a raíz de factores diversos, entre ellos, los históricos (conquista, dominación, colonialismo) o, incluso, los que responden a la lógica estructural: en este caso, el acento está puesto en "las condiciones formales del pasaje de un modo [de identificación] a otro por medio de una serie continua de variaciones intermedias..." (Descola 2014: 259). Explícitamente, Descola se esmeró por crear un "modelo de inteligibilidad de los hechos sociales y culturales que debía permanecer lo más neutro posible respecto de los presupuestos de nuestra propia ontología. Es por eso que esta ontología, el naturalismo, no es más que una de las cuatro variantes posibles en las maneras de objetivar el mundo" (2014: 235). El proyecto de Decola muestra precisamente que la modernidad es una formación ontológica particular entre otras que "distribuyen lo que existe y conciben sus relaciones constitutivas de un modo diferente" al modo en que lo hace la modernidad euro-occidental (Blaser 2009: 886).

Combinando las preocupaciones por las ontologías, la política y la no-humanidad, Mario Blaser (2009, 2013, 2016) propone la noción de conflictos ontológicos tras su experiencia etnográfica en el Chaco paraguayo. Su perspectiva abreva tanto en algunos desarrollos de la antropología francesa y brasileña como en discusiones propias de la academia norteamericana. En sus diversos trabajos, Blaser define "ontología" como el inventario de tipos de seres y sus relaciones posibles, expresa la centralidad de las prácticas que involucran a humanos y a no-humanos, y se interesa por las narraciones de sus interlocutores ishir del Chaco, pues en ellas se establece lo que existe y las relaciones. Este antropólogo argentino radicado en Canadá sostiene también la centralidad de "tomar en serio" a los no-humanos y dejar de considerarlos como metáforas de otras realidades, siguiendo los postulados de Viveiros de Castro. Su interés por la ontología lo condujo a preguntarse teóricamente por aspectos políticos, a partir de los cuales acuñó la noción de ontología política. En la medida en que los mundos son siempre el ensamblaje heterogéneo de humanos y no-humanos, Blaser indaga en qué es la política en estos ensamblajes que rompen con las dicotomías a las que estamos acostumbrados. 
Retoma la proposición cosmopolítica de Isabelle Stengers (2005) y expresa que, cuando los no-humanos no son concebidos como fruto de la imaginación, ellos entran en la escena política de nuestros interlocutores y, desde este punto de vista, ameritan ser considerados en una investigación antropológica. Blaser retoma la propuesta de Descola precisamente porque el antropólogo francés muestra la existencia de múltiples ontologías y porque da cuenta de que la ontología moderna es una entre otras que distribuye lo que existe de una forma diferente. El interés específico de Blaser (2013) será abordar este pluralismo ontológico desde los conflictos medioambientales, leídos como verdaderos conflictos ontológicos, ya que activan diversas formas de enactuar el mundo. Su acercamiento etnográfico al pluralismo y a los conflictos promueve "tomar 'las cosas' encontradas en el campo tal como se presentan en sí mismas, más que asumir que ellas significan, representan o abogan por otra cosa más" (Henare, Holbraad y Wastell 2007: 2).

Tanto Blaser como diversos autores interesados por las ontologías en variadas academias del mundo distinguen la proclamación de múltiples ontologías del postulado sobre la existencia de diferentes interpretaciones o epistemologías. Para ellos, lo que existe no serían ya diversas visiones de un mismo y único mundo, sino múltiples mundos y atribuciones diversas de propiedades a lo existente. Estas atribuciones diversas son las que marcan las diferencias ya no entre cosmovisiones o representaciones culturales, sino entre ontologías que coexisten, es decir, entre diversas maneras de mondier, de worlding o de componer los mundos.

Ahora bien, en estas coexistencias de ontologías se producen equívocos que Viveiros de Castro (2004) define como misunderstandings en el sentido de una falla en entender que los understandings, o comprensiones, no son compartidas por todos ni están relacionadas con formas imaginarias de ver el mundo, sino - como expresa también Blaser - con los mundos reales que son percibidos por diversos colectivos. Estas ideas antropológicas se anclan en el perspectivismo amerindio tal como fue conceptualizado por Viveiros de Castro (1996), es decir, remiten a la idea de que el mundo está compuesto por un conjunto de elementos cuya naturaleza varía de acuerdo al punto de vista del sujeto que percibe, punto de vista que se halla en el cuerpo. En este sentido, ya no se trataría de representaciones variables de un único mundo, sino de mundos diferentes, dado que todos los "existentes" perciben del mismo modo, o mediante las mismas categorías y valores, y lo que varía es el correlato objetivo que ellos ven. Tomadas como método antropológico, estas ideas amazónicas acerca de múltiples mundos a ser actualizados permitirían un acercamiento a la alteridad entendida como la expresión de puntos de vista plurales sobre mundos que también lo son (cf. Castro 2004).

Llevadas al análisis de las relaciones entre los pueblos indígenas y los Estados, estas ideas resultan productivas para analizar, por ejemplo, las múltiples 
reivindicaciones por las que los primeros se movilizan. Bien sabido es que, entre otros, en estos contextos suelen generarse conflictos y equívocos respecto del territorio, los recursos, la tierra; es decir, conflictos sobre los motivos por los cuales se lucha. En efecto, el territorio, la tierra y los recursos no remiten necesariamente a lo mismo para los Estados, las multinacionales y los pueblos indígenas. Esto no significa sólo que unos y otros tienen visiones diversas sobre el territorio al que se lo concibe, a fin de cuentas, como un mismo entorno ambiental. Sostener esta posición implicaría suponer que ese referente empírico que denominamos "territorio" (mundo, naturaleza, recursos) existe per se y es representado de maneras diferentes según las culturas. En cambio, las posturas diferentes respecto del territorio dan cuenta también de que las diferencias no son de orden cultural sino ontológico. Es decir, el foco se ubica en un plano anterior al de las representaciones culturales: aquel en el que se llevan a cabo las inferencias básicas acerca de lo que existe y lo que es un mundo. En este sentido, la ontología no tiene tanto que ver con una pluralidad de perspectivas culturales, sino con una multiplicidad de mundos. Esta postura admite la existencia de diferencias en las maneras de "enactuar" el mundo, de destacar elementos diferentes de lo perceptible. Si vamos a fondo en esta posición, tomar en serio estas otras maneras de componer el mundo es reconocer que no estamos hablando de epistemologías otras, sino de ontologías y, una vez reconocido esto, dejar entonces de pensar las ideas de los otros en términos de imaginación cultural, metáforas o símbolos de algo que nosotros conocemos de modo correcto. Los debates de Stengers sobre cosmopolítica llevados a la antropología interesada en los pueblos indígenas condujeron también a repensar los conceptos de política y de sujeto político para dichos pueblos. Una de las particularidades del giro ontológico fue la inversión entre conceptos analíticos y datos etnográficos: más que aplicar conceptos y categorías euro-americanas a las realidades de otros pueblos, algunos autores proponen que el objetivo de la disciplina es la conceptualización y que esto implica tomar las ideas del otro como conceptos. El mundo descripto por dichos conceptos será distinto al mundo euro-americano y, por ende, conceptos como "política" no remitirán necesariamente a lo mismo. La noción de política va de la mano de nociones particulares de persona y agencia. Entonces, la propuesta cosmopolítica incluye los planteos más generales del giro ontológico respecto de repensar la Gran División y las oposiciones de ella derivadas, así como la necesidad de reconocer las limitaciones de los conceptos euro-americanos. Las oposiciones naturaleza/política, sujeto/objeto, humano/no humano, identidad/alteridad, mito/historia, contaminación/pureza, entre otras, parecieran mostrarse incompatibles o remitir a cosas diferentes para las sociedades descritas por los artículos de este dossier.

Así como en el terreno de la política una aproximación ontológica permite pensar en términos pluriversales y ampliar lo que entendemos por política, 
el abordaje ontológico en el ámbito de la historia también se ha mostrado fructífero. En él, salta a la luz la dificultad de sostener la existencia de un único pasado, objetivo, circunscripto y externo, representado de modo diverso por cada cultura. En esta línea, el artículo de Sonia Sarra analiza el conflicto ontohistórico que surge de la confrontación entre el pasado chiriguano (antiguos guaraníes) reconstruido a partir de fuentes históricas y el pasado mítico narrado por los guaraníes contemporáneos de Jujuy. Si desde la historiografía la ocupación guaraní de las estribaciones surorientales de los Andes ocurrió en un tiempo posterior al poblamiento del continente y, aproximadamente, en fechas previas a la conquista, desde la perspectiva nativa la llegada guaraní a esta región tuvo lugar en un tiempo mítico y fue agenciada por mellizos míticos, antepasados no humanos de los guaraníes actuales. La pluralización de la historia no responde meramente a una inquietud intelectual, sino que, en el escenario capitalista de los ingenios azucareros en el cual los guaraníes de Jujuy se encuentran insertos, sus relatos míticos pueden constituirse como verdaderas herramientas de lucha por un territorio que les fue enajenado.

El territorio es el eje de análisis del artículo de Pedro Emilio Robledo incluido en este dossier. Allí, el autor aborda las equivocaciones que emergen cuando el modo de ocupar y transitar el territorio desde la perspectiva qom (tobas) del Chaco argentino se encuentra con la planificación del ordenamiento territorial gubernamental. Al etnografiar este contexto, Robledo describe rasgos de una ontología otra que se expresa, antes que en proposiciones, en trayectorias que acontecen entre el bosque y los parajes indígenas. Aquí encontramos ideas de humanidad y naturaleza que contrastan con aquellas que acompañan a los programas de desarrollo, tanto en su versión productivista como ecologista, donde la naturaleza se concibe bajo un control absoluto material y/o simbólico humano. Concretamente, el autor encuentra que en la experiencia de los qom el bosque circundante a sus moradas se presenta poblado de vida no humana caracterizada por la intencionalidad y normas sociales que recuerdan al modo como transcurre la vida en el mundo humano. Sin embargo, esta se desarrolla lejos del bosque, en asentamientos que, si bien permeados por pautas residenciales estatales, son los lugares donde se reproduce la vida humana a partir de la cohabitación, la comensalidad, pero también de afecciones chamánicas. En este contexto, habitar un territorio consiste en una práctica cosmopolítica que transcurre entre el bosque y los parajes indígenas, cuyas consecuencias afectan las capacidades de la persona y ello siempre se manifiesta en el cuerpo.

En el artículo de Gabriel Rodrigues Lopes el giro ontológico opera como un "arte del pharmakon" (Stengers 2017), es decir, como un remedio que advierte acerca del peligro de nombrar la diferencia en tanto malentendido epistémico. Más que un equívoco de orden epistemológico, la diferencia se funda en premisas ontológicas diversas a aquellas del antropólogo. A pesar de esto, su análisis del modo de existencia de los lapigados de la caatinga de 
Bahía (Brasil) nos confronta con el hecho de que además de remedio, el giro disciplinar puede ser también un veneno que se asocia "a un imperativo que siempre comisiona a portavoces privilegiados" (Stengers 2017: 137). Estos privilegiados difícilmente reconocen el hecho de que el hacer antropológico debe aún "desaprender" cierto método que lo funda; ese "cuerpo" que pretende dar sentido al sentido nativo. Entre los lapigados, la figura del Otro resulta central para proteger la virtualidad de un mundo posible. Dicho mundo se actualiza en la intensificación de las relaciones de personas "poderosas", con sus prácticas de curar, adivinar, "ver" y "virar", y con sus guías o espíritus aliados. El autor propone que la creatividad nativa no debe ser totalmente codificable por el antropólogo quien debería aspirar a dejarse guiar por los nativos, que prefieren hacer de su relación con el afuera uno de sus modos de pensar y acceder al conocimiento. La gramática estado-céntrica, nos muestra Rodrigues Lopez, interesada en determinar grupos, identidades, culturas o unidades políticas, amerita ser puesta en suspenso ante los "desconocidos" lapigados. Finalmente, su texto sostiene que la contra-antropología lapigada es una crítica a los fundamentos del "cuerpo" del antropólogo en la medida en que promueve una torsión del tomar en serio, propio del giro ontológico y permitir que "el mundo subjetivo de Otro afecte el modo de existir del antropólogo".

Una problematización similar en relación a los vínculos entre similitud y diferencia e identidad y alteridad recorre el artículo de Antonela dos Santos de este dossier. En él se analiza la voluntad de algunos ranqueles de la provincia de La Pampa (Argentina) de recuperar su "espiritualidad" en el marco de los procesos de reorganización y revisibilización étnica que este pueblo protagoniza desde hace unas décadas. A partir de etnografiar la multiplicidad, fragmentariedad y la convivencia de lógicas diversas que prevalecen entre los ranqueles de hoy en día, dos Santos propone pensar que el proceso de recuperación de dicha espiritualidad genera, tanto entre ranqueles como entre antropólogos, reflexiones que pueden ser consideradas del orden de lo ontológico. De hecho, mapear y caracterizar algunos posicionamientos indígenas en relación a este proyecto de recuperación conducen a la autora a, por un lado, constatar que los desacuerdos entre personas son inherentes a la variabilidad propia del mundo ranquel. Por otro lado, la llevan a sugerir que dichos desacuerdos producen, en ocasiones, fricciones ontológicas donde lo que está en juego es, justamente, el definir cuáles son los distintos existentes que pueblan el mundo ranquel en restauración, qué características tienen y cómo los ranqueles se relacionan con ellos. 


\section{BIBLIOGRAFÍA}

BLASER, Mario, 2009, "Political ontology: cultural studies without 'cultures'?", Cultural Studies, 23 (5-6): 873-896.

BLASER, Mario, 2013, "Ontological conflicts and the stories of peoples in spite of Europe: toward a conversation on political ontology", Current Anthropology, 54 (5): 547-568.

BLASER, Mario, 2016, "Is another cosmopolitics possible?", Cultural Anthropology, 31 (4): 545-570.

CASTRO, Eduardo Viveiros de, 1996, "Os pronomes cosmológicos e o perspectivismo ameríndio", Mana, 2 (2): 115-144.

CASTRO, Eduardo Viveiros de, 2004, "Perspectival anthropology and the method of controlled equivocation", Tipiti: Journal of the Society for the Anthropology of the Lowland South America, 2 (1): 3-22.

CHARBONNIER, Pierre, Gildas SALMON, y Peter SKAFISH, 2017, Comparative Metaphysics: Ontology after Anthropology. Lanham, Rowman \& Littlefield international.

DE LA CRUZ, Luis María, 2004, "El Estado y la cuestión de la tierra tras la frontera agropecuaria en Formosa: ¿geopolítica del desarrollo o del subdesarrollo?”, en Elena Belli, Ricardo Slavutsky y Héctor Hugo Trinchero (comps.), La Cuenca del Río Bermejo: Una Formación Social de Fronteras. Buenos Aires, Reunir, 221-267.

DESCOLA, Philippe, 2005, Par-delà nature et culture. París, Gallimard.

DESCOLA, Philippe, 2010, "Indigenous cosmopolitics in the Andes: conceptual reflections beyond 'politics'”, Cultural Anthropology, 25 (2): 334-370.

DESCOLA, Philippe, 2014, La Composition des mondes : Entretiens avec Pierre Charbonnier. París, Flammarion.

HENARE, Amiria, Martin HOLBRAAD, y Sari WASTELl, 2007, "Introduction: thinking through things”, en Amiria Henare, Martin Holbraad y Sari Wastell (comps.), Thinking through Things: Theorising Artefacts Ethnographically. Londres, Routledge, 1-31.

HOLBRAAD, Martin, 2007, "The power of powder: multiplicity and motion in the divinatory cosmology of Cuban Ifá (or mana, again)”, en Amiria Henare, Martin Holbraad y Sari Wastell (comps.), Thinking through Things: Theorising Artefacts Ethnographically. Londres, Routledge, 189-225.

HOLBRAAD, Martin, 2010, "Against the motion (2)", en Soumhya Venkatesan (comp.), "Ontology is just another word for culture: motion tabled at the 2008 meeting of the group for debates in anthropological theory, University of Manchester", Critique of Anthropology, 30 (2): 179-185.

HOLBRAAD, Martin, 2012. Truth in Motion: The Recursive Anthropology of Cuban Divination. Chicago, Chicago University Press.

HOLBRAAD, Martin, Morten PEDERSEN, y Eduardo Viveiros de CASTRO, 2014, "The politics of ontology: anthropological positions", Fieldsites, disponible en < http://culanth.org/ fieldsights/462-the-politics-of-ontology-anthropological-positions > (última consulta en junio de 2020).

IÑIGO CARRERA, Valeria, 2012, "Movilización indígena en el noreste formoseño: notas a propósito de un caso”, Relaciones de la Sociedad Argentina de Antropología, 37 (2): 457 -462 .

KOHN, Eduardo, 2012, "Proposal 1: anthropology beyond the human", Cambridge Anthropology, 30 (2): 136-146. 
KOHN, Eduardo, 2013, How Forests Think. Toward an Anthropology beyond the Human. Berkeley, CA, University of California Press.

LATOUR, Bruno, 1991, Nous n'avons jamais été modernes: Essais d'anthropologie symétrique. París, Editions La Découverte.

LATOUR, Bruno, 2013, Enquête sur les modes d'existence : Une anthropologie des Modernes. París, Editions La Découverte.

STENGERS, Isabelle, 2005, “The cosmopolitical proposal”, en Bruno Latour y Peter Weibel (comps.), Making Things Public: Atmospheres of Democracy. Cambridge, MA, The MIT Press, 994-1003.

STENGERS, Isabelle, 2017, En Tiempos de Catástrofe. Barcelona, Futuro Anterior Ediciones. VENKATESAN, Soumhya, 2010, "Ontology is just another word for culture: motion tabled at the 2008 meeting of the group for debates in anthropological theory, University of Manchester" Critique of Anthropology, 30 (2): 152-200. 